\title{
Precision Measurement of the Position-Space Wave Functions of Gravitationally Bound Ultracold Neutrons
}

\author{
Y. Kamiya, ${ }^{1}$ G. Ichikawa, ${ }^{2}$ and S. Komamiya ${ }^{1}$ \\ ${ }^{1}$ Department of Physics, Graduate School of Science, and International Center for Elementary Particle Physics, The University of Tokyo, \\ Tokyo 113-0033, Japan \\ ${ }^{2}$ Department of Physics, Nagoya University, Nagoya 464-8601, Japan
}

Correspondence should be addressed to Y. Kamiya; kamiya@icepp.s.u-tokyo.ac.jp

Received 12 June 2014; Accepted 26 August 2014; Published 10 September 2014

Academic Editor: Valery V. Nesvizhevsky

Copyright (C) 2014 Y. Kamiya et al. This is an open access article distributed under the Creative Commons Attribution License, which permits unrestricted use, distribution, and reproduction in any medium, provided the original work is properly cited. The publication of this article was funded by $\mathrm{SCOAP}^{3}$.

Gravity is the most familiar force at our natural length scale. However, it is still exotic from the view point of particle physics. The first experimental study of quantum effects under gravity was performed using a cold neutron beam in 1975. Following this, an investigation of gravitationally bound quantum states using ultracold neutrons was started in 2002. This quantum bound system is now well understood, and one can use it as a tunable tool to probe gravity. In this paper, we review a recent measurement of position-space wave functions of such gravitationally bound states and discuss issues related to this analysis, such as neutron loss models in a thin neutron guide, the formulation of phase space quantum mechanics, and UCN position sensitive detectors. The quantum modulation of neutron bound states measured in this experiment shows good agreement with the prediction from quantum mechanics.

\section{Introduction}

Phenomena due to the gravitational field have been well understood at the macroscopic scales. However, there are only a few cases of experiments at microscopic scales, due to gravity's extreme weakness when compared to the other forces, such as electromagnetic and nuclear forces.

Even though quantum mechanics was established in the early 1900s, the first experiment to investigate a quantum effect under gravity was reported in 1975 by the group of Colella [1], where a neutron interference pattern induced by a gravitational potential was observed. The major systematic uncertainty was attributed to a bending effect of an interferometer when one rotates the system to manipulate a relative quantum phase difference between two neutron paths. A subsequent series of experiments were carried out with careful studies of systematic effects by measuring the bending effect using $\mathrm{X}$-rays and considering the Sagnac effect [2] due to the rotation of the Earth [3, 4]. The latest attempt was performed using normal-symmetric and skewsymmetric interferometers and a two-wavelength difference measurement method. The agreement with theoretical calculation is at the $1 \%$ level [5].

Another approach to observe quantum effects under gravity was carried out by the group of Nesvizhevsky [6,7]. In this experiment, one measured a transmission of ultracold neutrons (UCNs) through a vertically thin neutron guide in the terrestrial gravitational field as a function of the slit thickness and showed the evidence of quantum bound states in the gravitational potential by analyzing the minimum height of a guide through which UCN can be transmitted. In a later detailed analysis, the characteristic sizes of positionspace wave functions for the first and second quantum states were evaluated with an uncertainty of around $10 \%$ [8]. The major systematic uncertainty in this evaluation came from the difficulty in modeling the rough surface of a scatterer used on the ceiling of the guide, which causes some deformation of the wave functions. To minimize the effects of the deformation, a "differential method" was designed, in which one directly measures the wave functions using a position sensitive detector. In this method, a neutron guide of moderate height can be used and the deforming effect 


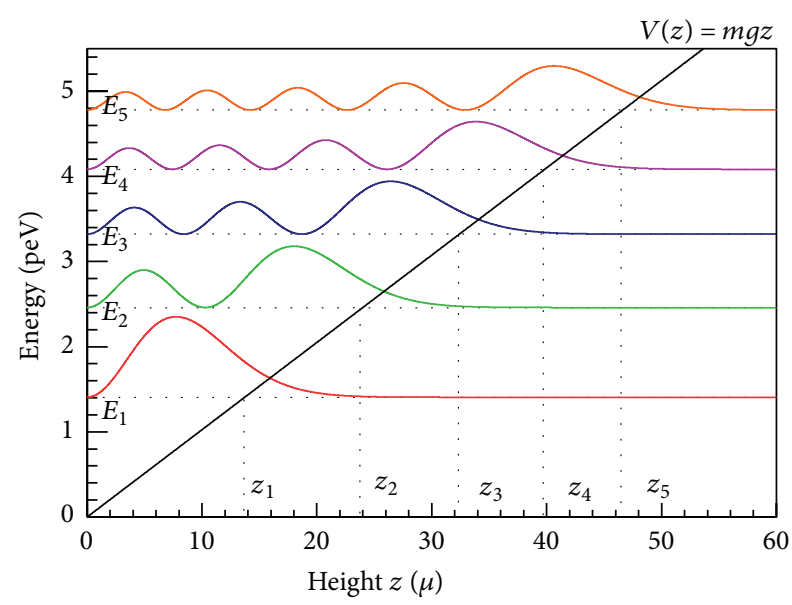

Figure 1: Probability distributions of gravitationally bound states of neutrons for the first five states [35]. Horizontal lines indicate eigenenergies $E_{n}$ and vertical lines show the classical turning points $z_{n}$ for each state.

was kept rather small compared to the previous "integrated method." The first attempt of the differential method was reported in [8], where a plastic nuclear track detector (CR39) with a uranium coating [9] was used as the detector.

These quantum systems, in which the UCN bounces on a smooth floor, are expressed by the time-independent Schrödinger equation

$$
\left\{-\frac{\hbar^{2}}{2 m} \frac{\mathrm{d}^{2}}{\mathrm{~d} z^{2}}+V(z)\right\} \psi_{n}(z)=E_{n} \psi_{n}(z)
$$

with the linear potential

$$
V(z)= \begin{cases}m g z, & z \geq 0 \\ \infty, & z \leq 0\end{cases}
$$

where $\psi_{n}$ and $E_{n}$ are the eigenfunctions and eigenenergies, $\hbar$ is the reduced Planck constant, $m$ is the neutron mass, and $g$ is the standard gravitational acceleration. Equation (1) can be rewritten in dimensionless form as

$$
\left(\frac{\mathrm{d}^{2}}{\mathrm{~d} \xi_{n}^{2}}-\xi_{n}\right) \psi_{n}\left(\xi_{n}\right)=0
$$

where $\xi_{n} \equiv z / z_{0}-E_{n} / E_{0}$ and the characteristic length and energy are given by

$$
\begin{gathered}
z_{0}=\left(\frac{\hbar^{2}}{2 m^{2} g}\right)^{1 / 3} \sim 6 \mu \mathrm{m}, \\
E_{0}=\left(\frac{m g^{2} \hbar^{2}}{2}\right)^{1 / 3} \sim 0.6 \mathrm{peV} .
\end{gathered}
$$

This is the Airy equation and solutions are described by Airy special functions, $\operatorname{Ai}\left(\xi_{n}\right)$ and $\operatorname{Bi}\left(\xi_{n}\right)$. Calculated probability distributions for the first five states are illustrated in Figure 1. Note that $\xi_{n}=0$, where $z_{n} \equiv z_{0} E_{n} / E_{0}$, are the classical turning points of a classical bouncing ball, which are denoted in the cross points of each eigenenergy and the potential line of $m g z$ in Figure 1.

This system is a suitable device with which to test the inverse square law of standard Newtonian gravity and to search for new gravity-like short-range interactions around these scales. The first limit to a non-Newtonian force with a Yukawa-type interaction potential using this microscopic system was reported in [10]. The limits for a CP-violating Yukawa-type potential were shown in [11, 12]. Several experimental schemes have been proposed to improve the sensitivity to such hypothetical new physics. One idea to achieve a better resolution on the measurement of the characteristic length is to utilize a convex reflection mirror to magnify the neutron distributions $[13,14]$. In this review, we discuss an experiment using this scheme to precisely measure the UCN position-space wave functions, performed by the group of Komamiya of the University of Tokyo [15]. Experiments exploiting other ideas for measuring the energy scale, that is, the energy differences between quantum states, are proposed by observing resonance transitions induced by a magnetic field [16-19] and mechanical vibrations [20]. These projects are called GRANIT [21] and qBounce, respectively. The first measurement of the resonance transition from the ground to the third state was reported by the group of Abele in [22]. Recently, a new limit for the CP-violating Yukawa-type potential using the resonance method was reported in [23]. It also shows a limit for the chameleon field [24-26], a dark energy candidate.

\section{Precision Measurement and Issues Related to This Analysis}

The precision measurement with a convex magnification mirror [15] was performed using a UCN source provided at ILL (Institut Laue-Langevin) [27]. The velocity distribution was measured by a TOF method and is well characterized by a Gaussian distribution with mean of $9.4 \mathrm{~m} / \mathrm{s}$ and standard deviation of $2.8 \mathrm{~m} / \mathrm{s}$. Figure 2(a) shows a schematic drawing of the experiment. The entire system was mounted on an antivibration table and magnetically shielded by a Permalloy sheet. The main components can be separated into three parts: a vertically thin collimating guide, a magnification mirror (rod), and a pixelated position sensitive detector. The layout of these components is shown in Figure 2(b).

2.1. Collimating Guide. The guide settles gravitationally bound quantum states. To clearly distinguish each quantum state, which has energy differences of order of $\Delta E \sim 1 \mathrm{peV}$, a resolving time of $\Delta t \sim \hbar / \Delta E \sim 1 \mathrm{msec}$ is required. Considering that the horizontal velocities of UCNs which we use are less than $15 \mathrm{~m} / \mathrm{s}$, the collimating guide was designed to have a sufficient length (192 $\mathrm{mm}$ in our setup) to form each quantum state. The bottom mirror is made of polished glass with roughness of $R_{a}$ (arithmetic average) $=0.03 \mu \mathrm{m}$. The ceiling scatterer is a Gd-Ti-Zr alloy (54/35/11) deposited on glass, with a Fermi potential tuned to be nearly zero and roughness of $R_{a}=0.4 \mu \mathrm{m}$ [28], which scatters out neutrons 


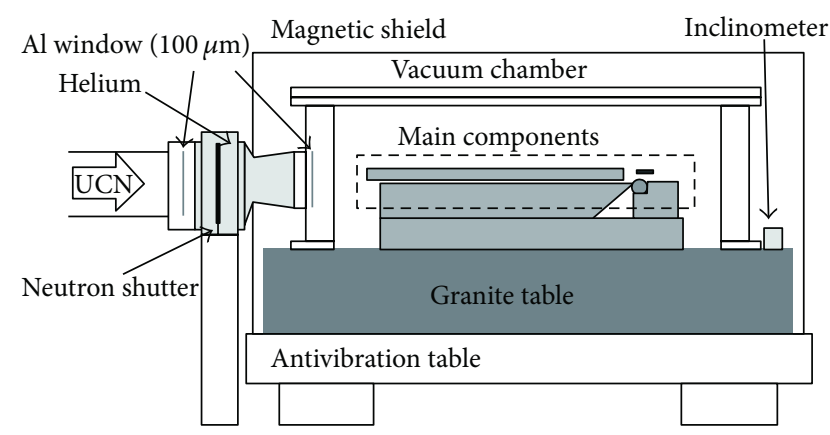

(a)

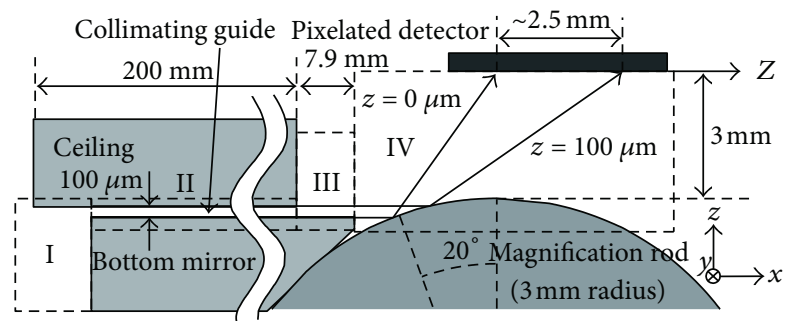

(b)

FIGURE 2: Schematic drawing of the precision measurement experiment using a convex magnification mirror [15]. (a) is an overview of the system and (b) shows the geometry of the main components.

in the higher states. The guide selects lower states with appropriate populations to improve the contrast of the quantum spatial modulation. (The eigenfunctions modulate somewhat coherently, especially around the lower region, except for the ground state, as seen in Figure 1. To improve the contrast of the quantum modulation, a method using a negative step of several tens of $\mu \mathrm{m}$, which transfers neutrons from the ground state to higher states, was considered in [9]. Results were reported in [29-31].) The neutron loss models for the scatterer or rough surface have been discussed in detail in [32-34], and they are still interesting issues not only for UCN guiding applications but also for storage experiments such as neutron EDM measurements. In the experiment reviewed in this paper, empirical models of the loss rates are adopted [15]. The loss rate by the scatterer, $\Gamma_{n}$, is assumed to be proportional to the probability of finding neutron in the roughness region and is given by

$$
\Gamma_{n}=\gamma \int_{h-2 \delta}^{h}\left|\widetilde{\psi}_{n}\right|^{2} \mathrm{~d} z,
$$

where $\gamma$ is a scaling constant, estimated from data to be $9.5_{-0.9}^{+0.7} \times 10^{4} \mathrm{~s}^{-1}, h$ is the height of the guide $(100 \mu \mathrm{m})$, $\delta$ is the roughness of the scatterer $(0.4 \mu \mathrm{m})$, and $\widetilde{\psi_{n}}$ are deformed wave functions in the guide. Neutron losses at the bottom mirror due to absorption, nonspecular reflection, upscattering, and other processes are modeled empirically as

$$
B_{n}=\beta \frac{g}{2 \sqrt{2}} \sqrt{\frac{m}{\widetilde{E_{n}}}},
$$

in which the loss rate is assumed to be proportional to the classical bouncing number per unit time, $g / 2 \widetilde{v}_{z, n \text {,max }}$, where $\beta$ is a scaling constant (estimated to be $0.38_{-0.03}^{+0.04}$ ), $\widetilde{v}_{z, n, \max } \equiv$ $\sqrt{2 \widetilde{E_{n}} / m}$ is the maximum vertical velocity of a neutron in the $n$th state, and $\widetilde{E_{n}}$ is the eigenenergy of the deformed wave functions. The transmissivity of the guide for each state can be written as

$$
\widetilde{p_{n}} \propto\left\langle\exp \left[-\frac{l}{v_{x}}\left(\Gamma_{n}+B_{n}\right)\right]\right\rangle_{v_{x}},
$$

where $\langle\cdots\rangle_{v_{x}}$ indicate the average over the neutron horizontal velocities. By applying a diabatic transition from region II to III, the population distribution (a probability in [15]) of neutrons for each state at the end of region III is estimated as in Figure 3(a).

2.2. Magnification Mirror. A Ni coated cylindrical rod is used as a magnifying convex mirror. Its radius is $3 \mathrm{~mm}$ and it is placed to have a grazing angle of $20 \mathrm{deg}$. for horizontally moving neutrons at the bottom floor level $(z=0)$. Figure 4 shows the magnification power as a function of the height [35]. It gives about 20 times magnification around the lower region of $z \sim 20 \mu \mathrm{m}$. Before depositing the Ni coating, the cylinder was polished at the Research Center for UltraPrecision Science and Technology, Osaka University. The roughness of the rod after the depositing was measured to be $R_{a}=1.9 \mathrm{~nm}$, two orders of magnitude smaller than the neutron wavelength.

To calculate the detected position distribution on the detector surface, the wave function at the end of the bottom floor is reexpressed by the Wigner phase space distribution $[36,37]$ :

$$
W\left(z, p_{z}\right) \equiv \frac{1}{2 \pi \hbar} \int_{-\infty}^{\infty} \delta \eta e^{-(i / \hbar) p_{z} \eta}\left\langle z+\frac{1}{2} \eta|\hat{\rho}| z-\frac{1}{2} \eta\right\rangle,
$$

where $p_{z}$ is the momentum and $\hat{\rho}$ is a density operator. The Wigner distribution is known as a phase space formulation of quantum mechanics and is widely used for quantum optics, for example, in the study of decoherence [38]. As an application to massive particles, one can find a paper which shows a phase space tomography of the Wigner distribution for a coherent atomic beam in a double-slit experiment [39]. Figure 3(b) shows the Wigner distribution constructed from the estimated populations in our experiment [15]. The time evolution of the Wigner distribution is calculated by the evolution of the density operator described by the Liouville-von Neumann equation

$$
\frac{\partial \hat{\rho}}{\partial t}=-\frac{i}{\hbar}[\widehat{H}, \widehat{\rho}]
$$




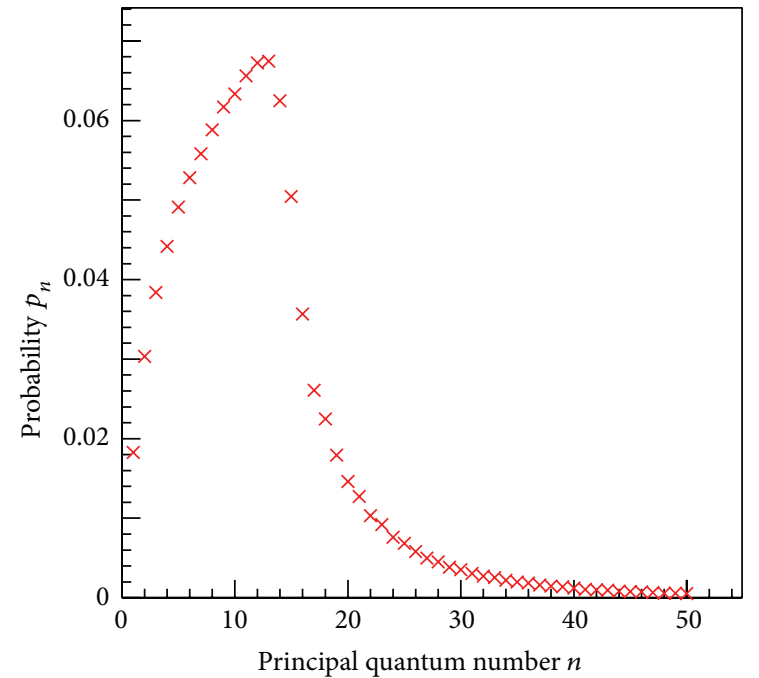

(a)

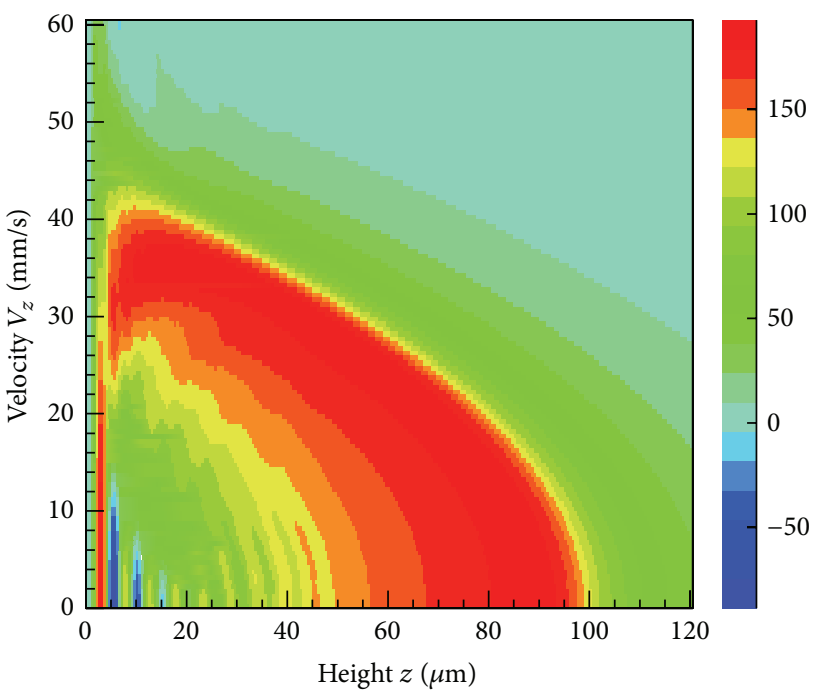

(b)

FIGURE 3: (a) is an estimated population distribution (probability distribution of the guide transmission) at the end of region III and (b) shows the corresponding Wigner phase space distribution [15].

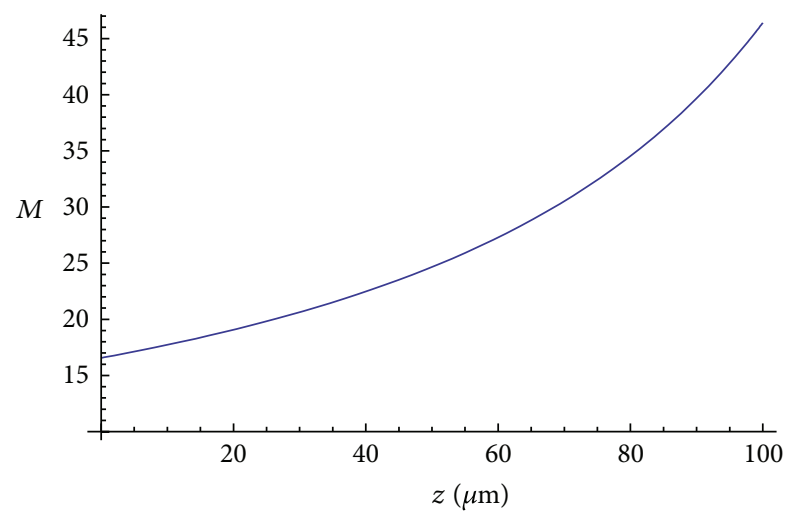

Figure 4: Magnification power $M$ of the cylindrical rod as a function of the height $z$ [35]. It is about 20 times magnification around the lower region.

where

$$
\widehat{H}=\frac{\widehat{p_{z}^{2}}}{2 m}+V(\widehat{z}) .
$$

By evaluating the kinetic part as

$$
T=-\frac{p_{z}}{m} \frac{\partial}{\partial z} W\left(z, p_{z}\right)
$$

and the potential part as

$$
\sum_{l=0}^{\infty} U_{l}=\sum_{l=0}^{\infty} \frac{(-1)^{l}(\hbar / 2)^{2 l}}{(2 l+1) !} \frac{\mathrm{d}^{2 l+1} V(z)}{\mathrm{d} z^{2 l+1}} \frac{\partial^{2 l+1}}{\partial p_{z}^{2 l+1}} W\left(z, p_{z}\right),
$$

one can obtainthe quantum Liouville equation for the Wigner distribution [40]

$$
\left(\frac{\partial}{\partial t}+\frac{p_{z}}{m} \frac{\partial}{\partial z}-\frac{\mathrm{d} V(z)}{\mathrm{d} z} \frac{\partial}{\partial p_{z}}\right) W\left(z, p_{z}\right)=\sum_{l=1}^{\infty} U_{l} .
$$

In the case of $V(z)=m g z$, the right-hand side of the equation vanishes and it becomes the classical Liouville equation. Therefore, in region IV, one can treat the evolution of each phase point of the Wigner distribution as a classical path under gravity. Figure 5 shows the measured data and the best theoretical estimation using this model. The corresponding $P$ value is 0.715 , and the experimental data support the quantum features described by the phase space formulation using the Wigner distribution [15].

2.3. Position Sensitive Detector for UCNs. A back-thinned CCD (HAMAMATSU S7030-1008) with thin $\mathrm{Ti}^{-}{ }^{10} \mathrm{~B}$-Ti layers is used for the position sensitive detector in the experiment [15]. Its pixel size is $24 \mu \mathrm{m} \times 24 \mu \mathrm{m}$ and the thickness of the active volume is about $20 \mu \mathrm{m}$. The ${ }^{10} \mathrm{~B}$ layer converts neutrons into charged particles by the nuclear reaction ${ }^{10} \mathrm{~B}(n, \alpha){ }^{7} \mathrm{Li}$. The secondary particles are emitted in a nearly back-to-back configuration. One of them deposits its kinetic energy in the active area and creates a charge cluster, which typically spreads into nine pixels. The weighted center of the charge cluster is a good estimation of the incident neutron position. The thicknesses of the layers are $20 \mathrm{~nm}$ and $200 \mathrm{~nm}$ for Ti and ${ }^{10} \mathrm{~B}$, respectively, and they are formed by evaporating directly on the CCD surface. The spatial resolution is measured to be $3.35 \pm 0.09 \mu \mathrm{m}$ by evaluating the line spread function (LSF) (see Figure 6) using very cold neutron beams at ILL [35]. For the details of the evaluation scheme, see [14, 41]. A neutron converter of ${ }^{6} \mathrm{Li}$ using ${ }^{6} \mathrm{Li}(n, \alpha)^{3} \mathrm{H}$ reaction was also investigated. It is concluded that the use of ${ }^{10} \mathrm{~B}$ gives better spatial resolution [41].

Other detectors using a silicon pixel device, Timepix [42], with ${ }^{6} \mathrm{LiF}$ and ${ }^{10} \mathrm{~B}$ converter were studied in [43]. Its pixel pitch is $55 \mu \mathrm{m} \times 55 \mu \mathrm{m}$ and the thickness of its silicon layer is about $300 \mu \mathrm{m}$. The spatial resolution for the ${ }^{6} \mathrm{LiF}$ converter was evaluated by LSF to be $2.3 \mu \mathrm{m}$, corresponding to about 


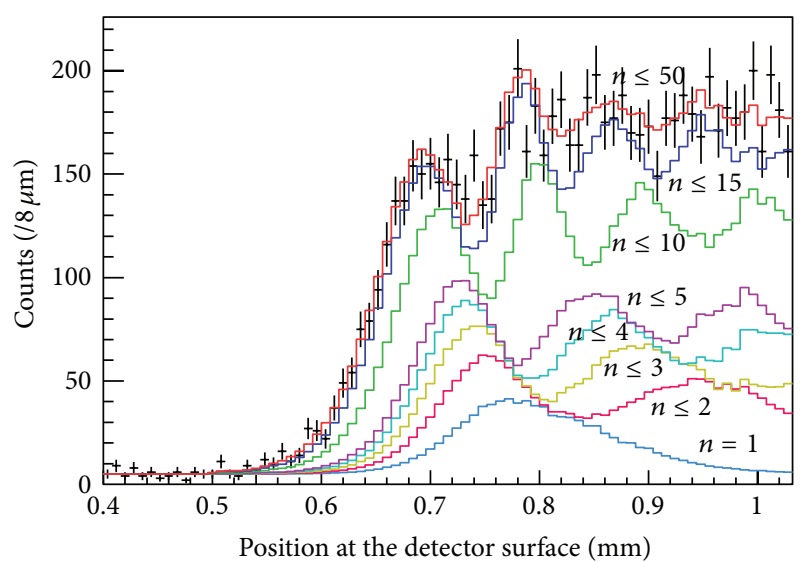

FIGURE 5: Black crosses show measured neutron position distribution [15]. Modulated distribution was clearly measured. The result shows good agreement with the quantum expectation calculated using phase space formalization by Wigner distribution $(P$ value is $0.715)$. The best estimated line is shown by a red solid curve and the other lines indicate contributions of each state to the distribution. For definitions of fitting parameters, best fit values, systematic uncertainties, and the other details, see [15].

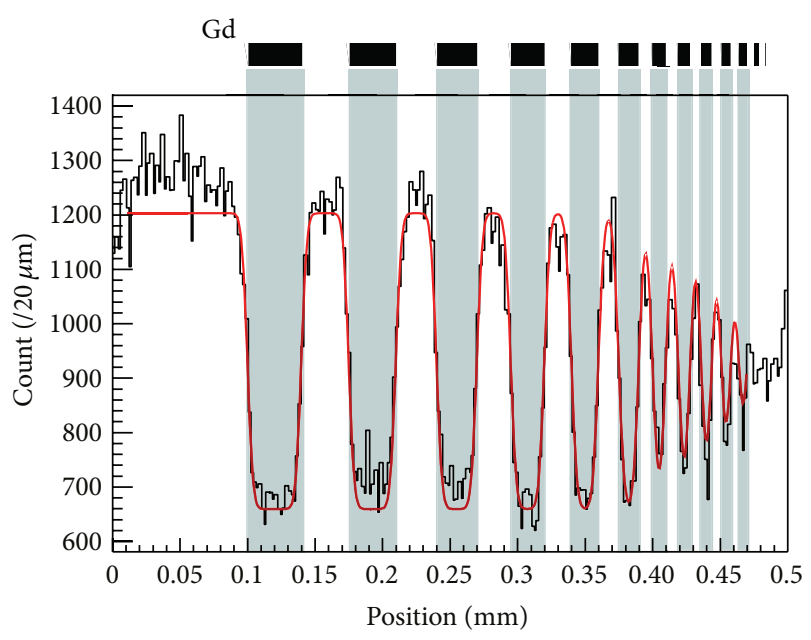

FIGURE 6: Fitting results of a Gd shadow pattern [35]. Spatial resolution is measured to be $3.35 \pm 0.09 \mu \mathrm{m}$ by evaluating these line spread functions. For details of evaluation method, see [14, 41].

$5.3 \mu \mathrm{m}$ in FWHM of the point spread function (PSF). The performance of the ${ }^{10} \mathrm{~B}$ converter is estimated by MonteCarlo simulations to be better than $3 \mu \mathrm{m}$ in FWHM of the PSF.

Another detector concept of uranium coated plastic nuclear tracker (CR39) [9] was used in several experiments $[8,29-31,44]$. Usually two fission fragments are emitted from a thin ${ }^{235} \mathrm{U}$ coating, and one of the daughter nuclei makes a track of defects in the CR39. By chemical etching, the diameter of track points is increased up to $1 \mu \mathrm{m}$, allowing us to scan these vertexes using an optical microscope. Position resolution is around $1 \mu \mathrm{m}$ [9]. By carefully analyzing the vertex shape, the spatial resolution can be improved to $0.7 \mu \mathrm{m}$ [44].

\section{Summary}

The quantum system of a gravitationally bound neutron is one of the most suitable tools to investigate gravity or gravitylike hypothetical interactions around the scale of $10 \mu \mathrm{m}$ in length or $1 \mathrm{peV}$ in energy. After establishing this research field by the pioneering work in observation of the quantum state [6], experimental schemes for precision measurements of these characteristic scales have developed rapidly, and nowadays one can establish limits for parameter spaces of new physics experimentally $[10-12,23]$. Furthermore one can start discussing a phase space formulation of quantum physics for the gravitationally bound quantum state [15]. An experiment for a possible test of phase space formalization using spatial interference is under preparation.

\section{Conflict of Interests}

The authors declare that there is no conflict of interests regarding the publication of this paper.

\section{Acknowledgments}

The authors would like to thank Valery V. Nesvizhevsky (Institut Laue-Langevin), Hartmut Abele (Vienna University of Technology), William Snow (Indiana University), Peter Geltenbolt (Institut Laue-Langevin), and all participants in the GRANIT-2014 Workshop for interesting discussions and helpful suggestions. This material is based upon work supported by JSPS KAKENHI Grants no. 20340050 and no. 24340045 and Grant-in-Aid for JSPS Fellows no. 22.1661.

\section{References}

[1] R. Colella, A. W. Overhauser, and S. A. Werner, "Observation of gravitationally induced quantum interference," Physical Review Letters, vol. 34, no. 23, pp. 1472-1474, 1975.

[2] J. J. Sakurai, "Comments on quantum-mechanical interference due to the Earth's rotation," Physical Review D, vol. 21, Article ID 2993, 1980.

[3] J.-L. Staudenmann, S. A. Werner, R. Colella, and A. W. Overhauser, "Gravity and inertia in quantum mechanics," Physical Review A, vol. 21, no. 5, pp. 1419-1438, 1980.

[4] S. A. Werner, H. Kaiser, M. Arif, and R. Clothier, "Neutron interference induced by gravity: New results and interpretations," Physica B, vol. 151, no. 1-2, pp. 22-35, 1988.

[5] K. C. Littrell, B. E. Allman, and S. A. Werner, "Two-wavelengthdifference measurement of gravitationally induced quantum interference phases," Physical Review A, vol. 56, article 1767, 1997.

[6] V. V. Nesvizhevsky, H. G. Börner, A. K. Petukhov et al., "Quantum states of neutrons in the Earth's gravitational field," Nature, vol. 415, no. 6869, pp. 297-299, 2002.

[7] V. V. Nesvizhevsky, H. G. Börner, A. M. Gagarski et al., "Measurement of quantum states of neutrons in the Earth's gravitational field," Physical Review D, vol. 67, Article ID 102002, 2003. 
[8] V. V. Nesvizhevsky, A. K. Petukhov, H. G. Börner et al., "Study of the neutron quantum states in the gravity field," The European Physical Journal C, vol. 40, no. 4, pp. 479-491, 2005.

[9] V. V. Nesvizhevsky, H. Börner, A. M. Gagarski et al., "Search for quantum states of the neutron in a gravitational field: gravitational levels," Nuclear Instruments and Methods in Physics Research A, vol. 440, no. 3, pp. 754-759, 2000.

[10] V. V. Nesvizhevsky and K. V. Protasov, "Constraints on nonNewtonian gravity from the experiment on neutron quantum states in the earth's gravitational field," Classical and Quantum Gravity, vol. 21, no. 19, pp. 4557-4566, 2004.

[11] S. Baeßler, V. V. Nesvizhevsky, K. V. Protasov, and A. Y. Voronin, "A new constraint for the coupling of axion-like particles to matter via ultra-cold neutron gravitational experiments," Physical Review D, vol. 75, Article ID 075006, 2007.

[12] S. Baeßlera, V. V. Nesvizhevskyb, G. Pignol et al., "Constraints on spin-dependent short-range interactions using gravitational quantum levels of ultracold neutrons," Nuclear Instruments and Methods in Physics Research Section A: Accelerators, Spectrometers, Detectors and Associated Equipment, vol. 611, pp. 149-152, 2009.

[13] V. V. Nesvizhevsky, Preprint ILL 96NE14T, 1994.

[14] T. Sanuki, S. Komamiya, S. Kawasaki, and S. Sonoda, "Proposal for measuring the quantum states of neutrons in the gravitational field with a CCD-based pixel sensor," Nuclear Instruments and Methods in Physics Research A, vol. 600, pp. 657-660, 2009.

[15] G. Ichikawa, S. Komamiya, Y. Kamiya et al., "Observation of the spatial distribution of gravitationally bound quantum states of ultracold neutrons and its derivation using the wigner function," Physical Review Letters, vol. 112, Article ID 071101, 2014.

[16] P. Schmidt-Wellenburg, K. H. Andersena, P. Courtois et al., "Ultracold-neutron infrastructure for the gravitational spectrometer GRANIT," Nuclear Instruments and Methods in Physics Research Section A, vol. 611, no. 2-3, pp. 267-271, 2009.

[17] M. Kreuz, V. V. Nesvizhevsky, P. Schmidt-Wellenburg et al., "A method to measure the resonance transitions between the gravitationally bound quantum states of neutrons in the GRANIT spectrometer," Nuclear Instruments and Methods in Physics Research Section A: Accelerators, Spectrometers, Detectors and Associated Equipment, vol. 611, no. 2-3, pp. 326-330, 2009.

[18] S. Baeßler, M. Beau, GRANIT Collaboration et al., "The GRANIT spectrometer," Comptes Rendus Physique, vol. 12, pp. 707-728, 2011.

[19] V. V. Nesvizhevsky, "Gravitational quantum states of neutrons and the new granit spectrometer," Modern Physics Letters A, vol. 27, Article ID 1230006, 2012.

[20] H. Abele, T. Jenke, H. Leeb, and J. Schmiedmayer, "Ramsey's method of separated oscillating fields and its application to gravitationally induced quantum phaseshifts," Physical Review $D$, vol. 81, Article ID 065019, 2010.

[21] D. Roulier, F. Vezzu, S. Baessler et al., "Status of the GRANIT facility," Advances in High Energy Physics, vol. 2014, Article ID 730437, 2014.

[22] T. Jenke, P. Geltenbort, H. Lemmel, and H. Abele, "Realization of a gravity-resonance-spectroscopy technique," Nature Physics, vol. 7, no. 6, pp. 468-472, 2011.

[23] T. Jenke, G. Cronenberg, J. Burgdorfer et al., "Gravity resonance spectroscopy constrains dark energy and dark matter scenarios," Physical Review Letters, vol. 112, Article ID 151105, 2014.
[24] J. Khoury and A. Weltman, "Chameleon fields: awaiting surprises for tests of gravity in space," Physical Review Letters, vol. 93, no. 17, Article ID 171104, 2004.

[25] D. F. Mota and D. J. A. Shaw, "Strongly coupled chameleon fields: new horizons in scalar field theory," Physical Review Letters, vol. 97, Article ID 151102, 2006.

[26] J. Khoury, "Chameleon field theories," Classical and Quantum Gravity, vol. 30, Article ID 214004, 2013.

[27] A. Steyeri, H. Nagel, F. X. Schreiber et al., "A new source of cold and ultracold neutrons," Physics Letters A, vol. 116, pp. 347-352, 1986.

[28] Y. Kamiya, S. Kawasaki, G. Ichikawa et al., "Development of the CCD-based Detector with Fine Spatial Resolution," KURRI Progress Report CO1-1, 2008, http://www.rri.kyotou.ac.jp/PUB/report/PR/ProgRep2008/CO1.pdf.

[29] V. V. Nesvizhevsky, H. G. Borner, A. K. Retoukhov et al., "Quantum falling in the gravitational field," ILL Annual Report, Institut Laue-Langevin, 2004, http://www.ill.eu/quick-links/ publications/annual-report/.

[30] H. Abele, T. Jenke, D. Stadler, and P. Geltenbort, "QuBounce: the dynamics of ultra-cold neutrons falling in the gravity potential of the Earth," Nuclear Physics A, vol. 827, pp. 593c-595c, 2009.

[31] T. Jenke, D. Stadler, H. Abele, and P. Geltenbort, "QBOUNCE-experiments with quantum bouncing ultracold neutrons," Nuclear Instruments and Methods in Physics Research A, vol. 611, no. 2-3, pp. 318-321, 2009.

[32] A. E. Meyerovich and V. V. Nesvizhevsky, "Gravitational quantum states of neutrons in a rough waveguide," Physical Review A, vol. 73, Article ID 063616, 2006.

[33] A. Y. Voronin, H. Abele, S. Baeßler et al., "Quantum motion of a neutron in a waveguide in the gravitational field," Physical Review D, vol. 73, Article ID 044029, 2006.

[34] A. Westphal, H. Abele, S. Baeßler, V. V. Nesvizhevsky, K. V. Protasov, and A. Y. Voronin, "A quantum mechanical description of the experiment on the observation of gravitationally bound states," The European Physical Journal C, vol. 51, pp. 367-375, 2007.

[35] G. Ichikawa, Observation of gravitationally bound quantum states of Ul-tracold neutrons using a pixelated detector $[P h D$. dissertation], The University of Tokyo, 2013.

[36] E. Wigner, "On the quantum correction for thermodynamic equilibrium," Physical Review, vol. 40, no. 5, pp. 749-759, 1932.

[37] M. Hillery, R. F. O'Connell, M. O. Scully, and E. P. Wigner, "Distribution functions in physics: fundamentals," Physics Reports, vol. 106, no. 3, pp. 121-167, 1984.

[38] L. Mandel and E. Wolf, Optical Coherence and Quantum Optics, Cambridge University Press, 1995.

[39] C. Kurtsiefer, T. Pfau, and J. Mlynek, "Measurement of the wigner function of an ensemble of helium atoms," Nature, vol. 386, no. 6621, pp. 150-153, 1997.

[40] W. P. Schleich, Quantum Optics in Phase Space, Wiley-VCH, 2001.

[41] S. Kawasaki, G. Ichikawa, M. Hino et al., "Development of a pixel detector for ultra-cold neutrons," Nuclear Instruments and Methods in Physics Research A, vol. 615, no. 1, pp. 42-47, 2010.

[42] X. Llopart, R. Ballabriga, M. Campbell, L. Tlustos, and W. Wong, "Timepix, a $65 \mathrm{k}$ programmable pixel readout chip for arrival time, energy and/or photon counting measurements," Nuclear Instruments and Methods in Physics Research A: Accelerators, Spectrometers, Detectors and Associated Equipment, vol. 581, pp. 485-494, 2007. 
[43] J. Jakubek, P. Schmidt-Wellenburg, P. Geltenbort et al., "A coated pixel device TimePix with micron spatial resolution for UCN detection," Nuclear Instruments and Methods in Physics Research A, vol. 600, no. 3, pp. 651-656, 2009.

[44] T. Jenke, G. Cronenberg, H. Filter et al., "Ultracold neutron detectors based on ${ }^{10} \mathrm{~B}$ converters used in the qBounce experiments," Nuclear Instruments and Methods in Physics Research Section A, vol. 732, pp. 1-8, 2013. 

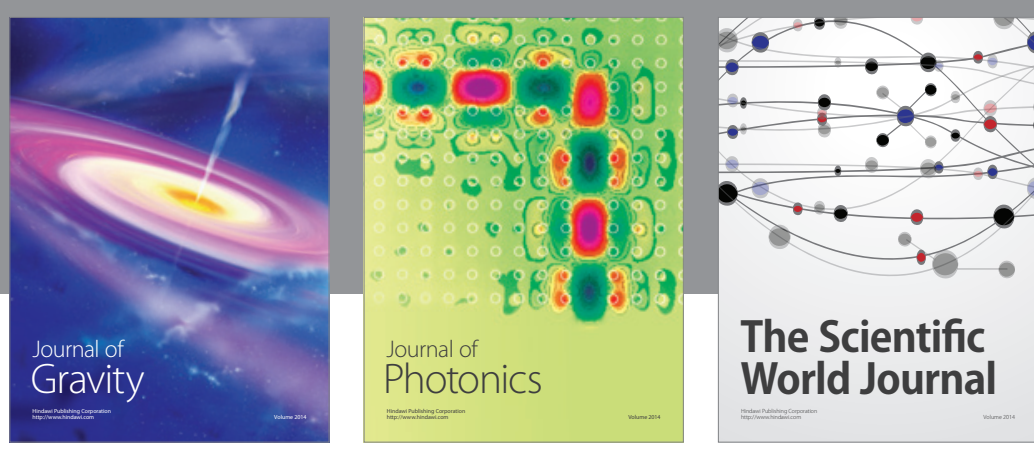

The Scientific World Journal
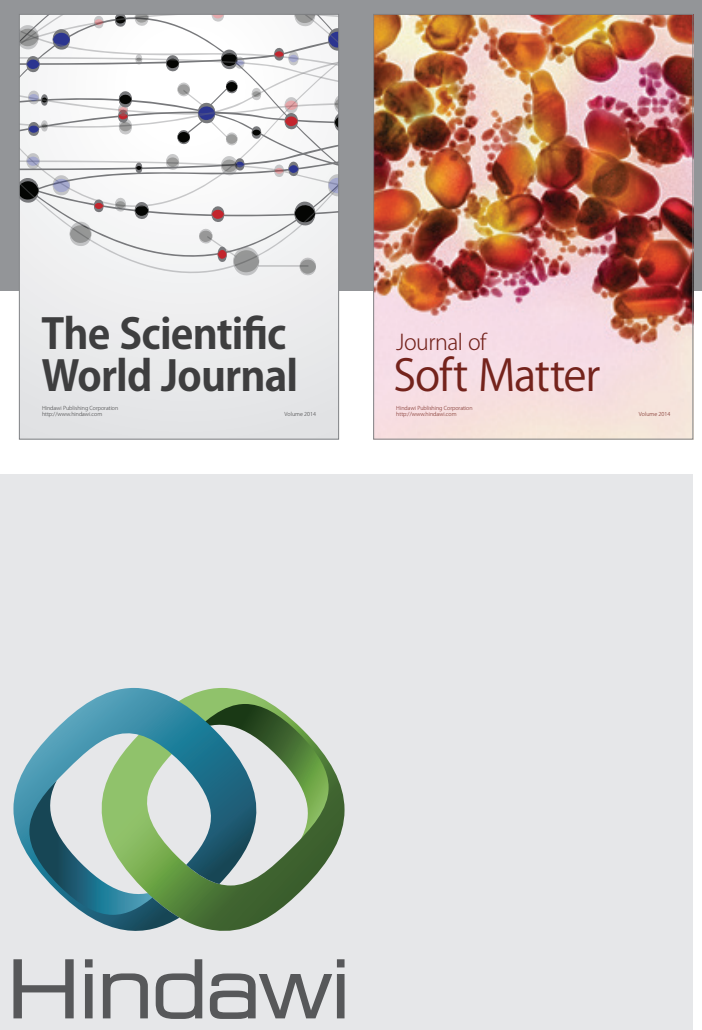

Submit your manuscripts at

http://www.hindawi.com

nternational Journal of

Statistical Mechanics
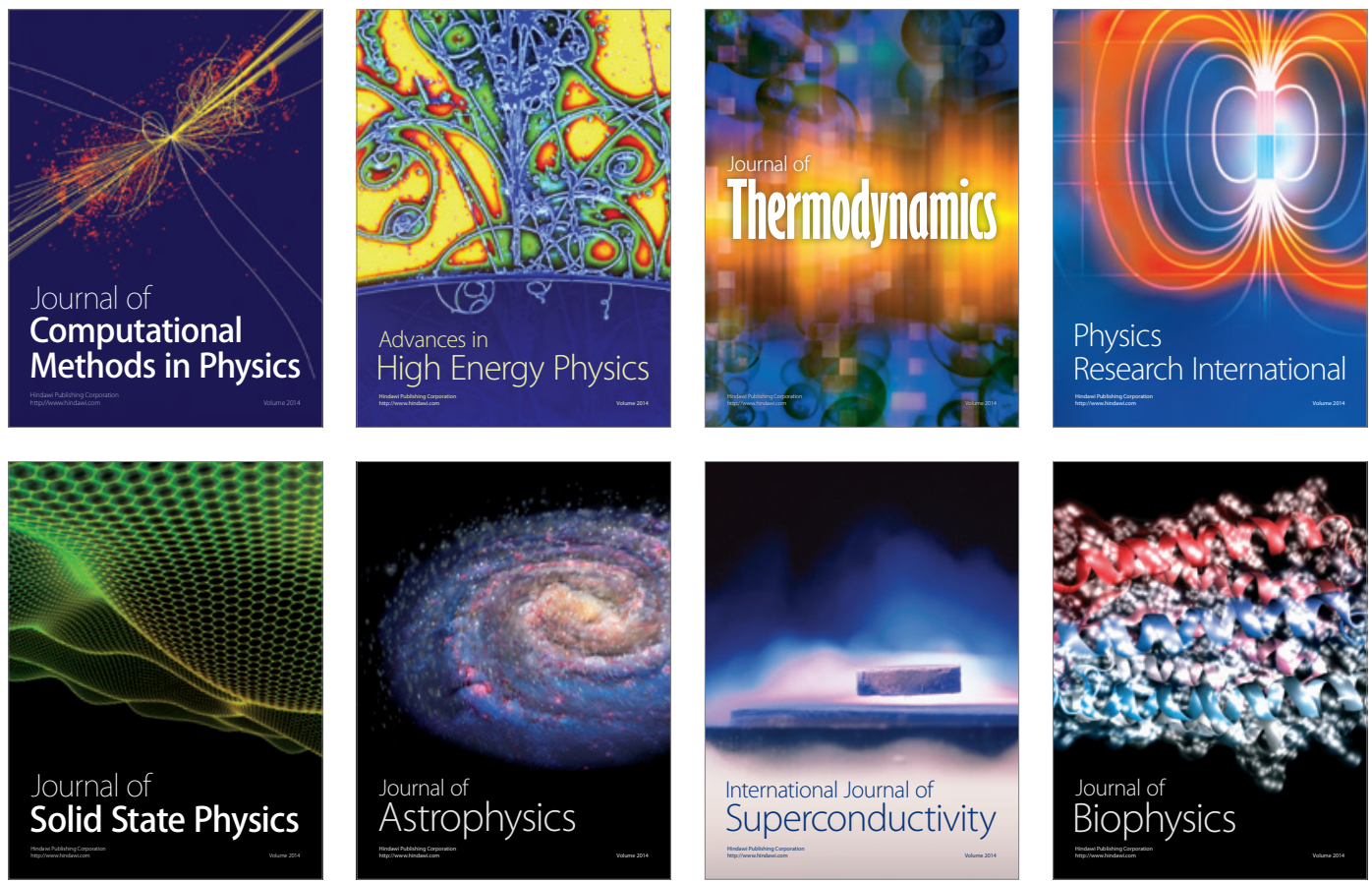
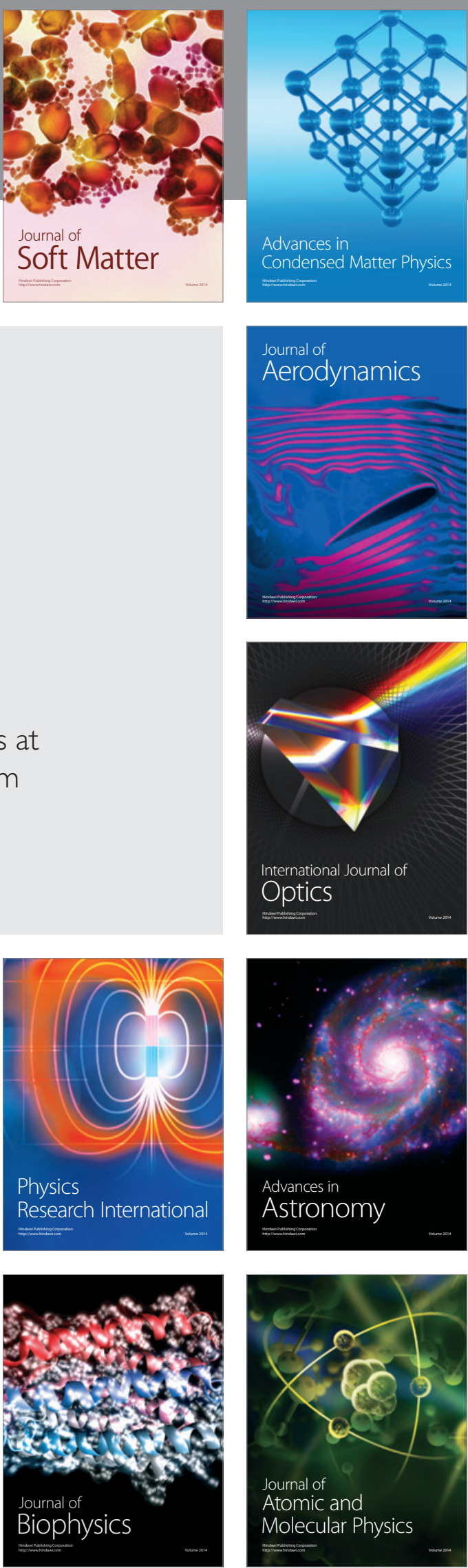